\title{
Optimizing Experimental Parameters and Lateral Resolution in LP-EM for Imaging Low-Z Colloids Using Monte Carlo Simulations
}

\author{
$\underline{\text { Peter Kunnas }}^{1}$ and Niels de Jonge ${ }^{2,3}$ \\ ${ }^{1}$ Faculty of Physics, University of Vienna, Vienna, Austria. \\ ${ }^{2}$ Leibniz Institute for New Materials, Saarbrücken, Germany. \\ ${ }^{3}$ Department of Physics, Saarland University, Saarbrücken, Germany.
}

To maximize the amount of data obtained from an experiment involving beam-sensitive material, it is helpful to optimize the imaging parameters of the electron microscope [1]. Optimal imaging settings for imaging low-atomic number $(\mathrm{Z})$ materials in liquid, ice, and related systems have been studied using analytical [2, 3], experimental [4], and simulated approaches [5, 6]. In some cases, simulated image data has also been used to support experimental findings in liquid-phase electron microscopy (LP-EM) via scanning transmission electron microscopy (STEM) [7]. We used Monte Carlo (MC) simulation software (Casino) $[6,8]$ for estimating the image formation in liquid samples, and to optimize an LP-EM system to visualize low-Z, hierarchical colloidal assemblies that consisted of oppositely charged, $30 \mathrm{~nm}$ diameter, amino-functionalized silica nanoparticles (SiONPs) spontaneously forming a shell around $100 \mathrm{~nm}-$ diameter, negatively charged polystyrene microspheres (PMs) [9]. The PMs consisted of a polymeric material whose structures were affected by the electron beam $[10,11]$. SiONPs have been found to change shape [12], and agglomerate [13] under the electron beam.

An example of the physical three-dimensional (3D) model used for the MC simulations is presented in Figure 1A. The topic of interest was how variations in the dark-field (DF) collection angle $(\beta)$, the probe convergence angle $(\alpha)$, the electron dose $\left(D_{e}\right)$, and liquid thickness $(t)$ affect the visibility, signal-to-noise ratio $(S N R)$, and resolution $d$ of low-Z nano-objects in liquid. The resulting 32-bit images of size 120x107 pixels were cropped, downsampled to 8-bit, and normalized, and then presented with comparable average intensity levels, as shown in Figure 1B. The normalization was done with the "Enhance Contrast" function in ImageJ software ( $0.3 \%$ of the pixels were allowed to become oversaturated). A relation was derived to describe the distinguishability for a multipixel object via Rose-criterion so that an object with $S N R_{L}{ }^{*} \geq 3$ is still visible as indicated in Figure 1C.Visual inspection of the data indicated that increasing $D_{e}$ and decreasing $t$ consistently improved the visibility of nano-objects against the liquid background. In the case of SiONP, the $D_{e}=2.1 \mathrm{e}^{-} \AA^{-2}$ was sufficient to resolve the object from the background for most of the tested settings. The PM showed an inversion of contrast at low values of $\beta$. In order to evaluate the predicted image quality while changing the microscope settings and $t$, the simulated image data were analyzed as shown in Figure 1C. The $S N R$ decreased with increasing t due to increased electron scattering while the electrons passed the sample. However, in most of the plots, the decrease of $S N R$ was not monotonous. Rather a plateau was visible below and above $t=300-500 \mathrm{~nm}$, which would suggest that keeping $t<300 \mathrm{~nm}$ is important for achieving the best resolution.

\section{References:}

[1] N de Jonge et al, Nature Reviews Materials 4 (2019), p. 61.

[2] DF Parsons, Advances in Biological and Medical Physics 15 (1974), p. 161.

[3] N de Jonge, Ultramicroscopy 187 (2018), p.113.

[4] TH Moser et al, Micron 117 (2019), p. 8 
[5] K Gnanasekaran, G de With and H Friedrich, Royal Society Open Science 5 (2018), p. 171838.

[6] H Demers et al, Microscopy and microanalysis 16 (2010), p. 795.

[7] TAJ Welling et al, Particle \& Particle Systems Characterization (2020), p. 2000003.

[8] P Hovington et al, Scanning 19 (1997), p. 1.

[9] MA Moradi et al, Nature Materials 20 (2021), p. 541.

[10] S Azim et al, Microscopy and Microanalysis (2020), p. 1.

[11] S Kitching and AM Donald, Journal of Microscopy 190 (1998), p. 357.

[12] J Zečević et al, Small 13 (2017), p. 1602466.

[13] MWP van de Put et al, Small 11 (2015), p. 585.

[14] The authors thank E. Arzt for his support through INM.

a)

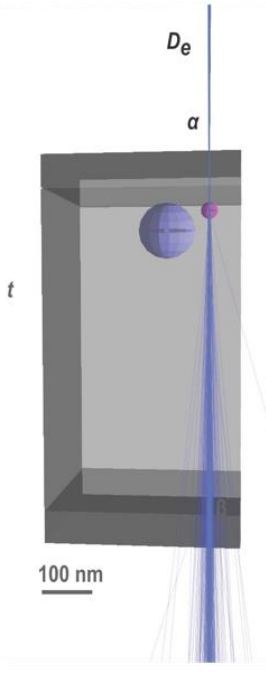

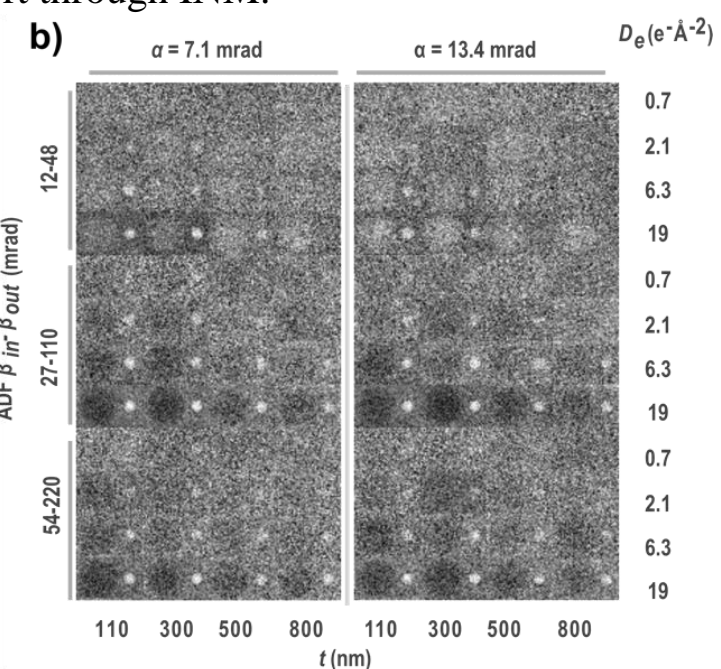

c)

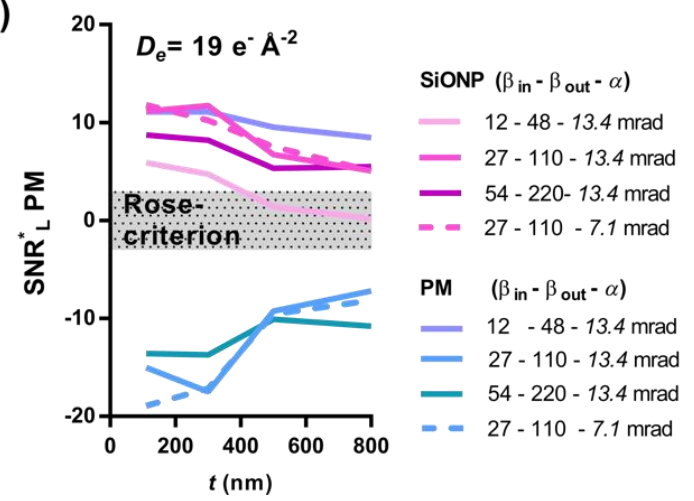

Figure 1. Monte Carlo simulation of liquid-phase STEM. A) A screen capture of the side view of the modeled liquid cell where a $100 \mathrm{~nm}$-diameter (PM) and a $30 \mathrm{~nm}$-diameter SiONP are positioned at the upper silicon nitride membrane of thickness $t_{S i N}=50 \mathrm{~nm}$. The liquid's $t=500 \mathrm{~nm}$. Trajectories of 24947 simulated electrons are shown in blue that correspond to $D_{e}=19 \mathrm{e}^{-} \AA^{-2}$ for the pixel size $s=3.7 \mathrm{~nm}$. B) Simulated image for a total of 96 tested combinations for the darkfield (DF) detector settings with innerand outer detector semi-angles $\beta_{\text {in }}$ and $\beta_{\text {out }}$, resp., and beam acceptance semi-angle $\alpha$. PM was located on the left side of each sub-panel, and SiONP was located on the right side. C) Quantitative comparison between STEM conditions where the SNR and visibility of PM and SiONP were determined for the simulated image of panel B. The obtained $S N R$ was plotted against the $t$ for the tested values of $\alpha$ and $\beta$. 